\section{OPEN PAEDIATRIC INTENSIVE CARE UNITS: STRENGTHS AND WEAKNESSES}

\author{
A. Bagnasco, M. Costa, L. Sasso \\ Università di Genova, Genoa, Italy
}

Aim: Reducing stress factors of parents, children and health care staff in open paediatric intensive care units (PICUs).Backgroundln Italy, there are 303 Intensive Care Units (ICUs), of these 99\% have very stringent policies for visitors. In literature, it is reported that open PICUs have the potential to reduce stress factors in patients and their families. Some studies found that allowing parents to stay overnight in the paediatric intensive care unit reduced the level of stress in both parent and child.

Materials and Methods: Through the Focus Group with nurses, we obtained data that were then processed and included into separate tables for parents, patients and nurses, highlighting the strengths and the weaknesses, as well as the number of interventions for each category.

Results: The three main categories of answers were obtained: 1) agreement with open PICUs (56\%); 2) positive answers for parents staying next to their children overnight $(75 \%) ; 3$ ) during emergency procedures parents often interfered and this was seen as a weakness by nurses ( $80 \%)$.

Conclusions: The presence of parents in the PICU was mainly judged positively both by nurses and parents, although some nurses claimed there were some weaknesses linked to the quality of the healthcare procedures. Another weakness was the interference of emotionally involved parents during emergency procedures.To achieve the desired outcomes and changes, we would suggest specific training sessions, briefings, improved facilities and psychological support for the staff.

Keywords: Open PICUs, stress factors, nurses, parents, children, reorganization

\section{9}

\section{TEMPOROMANDIBULAR JOINT DISORDER IN CHILDREN AND ADOLESCENTS COMPARED WITH ADULT PATIENT POPULATION}

\author{
T. Badel ${ }^{1}$, M. Marotti² I. Savić Pavicin ${ }^{3}$,
} D. Podoreški ${ }^{2}$, J. Kern ${ }^{4}$

${ }^{1}$ Department of Prosthodontics, School of Dental Medicine, ${ }^{2}$ Department of Diagnostic and Interventional Radiology, Clinical Hospital 'Sestre Milosrdnice', 'BDepartment of Dental Anthropology, School of Dental Medicine, ${ }^{4}$ Department of Medical Statistics, Epidemiology and Medical Informatics, School of Public Health "Andrija Štampar", School of Medicine, Zagreb, Croatia

Background and aims: The aim of this study was to compare clinical characteristics of children and adolescents in the sample of general patient population with temporomandibular joint (TMJ) disorders.

Methods: A prospective study was carried out between January 2001 and December 2008 and it included 110 patients with anterior disc displacement of TMJ. The whole sample of patients was divided in two groups: 16 patients aged under 20 and the remaining 94 patients (ranging from 20-82) who were seeking treatment. Diagnostics was based on clinical examination and it was confirmed by magnetic resonance imaging of all patients' TMJs. Pain intensity was rated on a visual-analogue scale (AVS 1-10).

Results: There is no statistical difference between average pain in patients over 20 (5.72) and patient aged up to 19 (4.96) evaluated by AVS ( $p=0.156)$. There was a statistically significant difference $(p=0.002)$ in pain duration: patients over 20 years of age reported shorter duration of experienced pain (10.57 months) than patients aged up to 19 (5.5 months). There is no difference between average active mouth opening in patients over $20(41.57 \mathrm{~mm})$ and patients aged up to $19(39.19 \mathrm{~mm})(p=0.303)$. The main symptom in both groups, patients over $20(79.79 \%)$ and patients up to 19 years of age $(56.25 \%)$, was TMJ pain $(p=0.128)$.

Conclusions: Incidence of TMJ disorders is most frequent between 18 and 40 years of age, however, in this study it was found that $14.55 \%$ were patients aged over 20. They experienced shorter TMJ pain duration before seeking treatment. 